\title{
Box diagram contribution to the axial two-nucleon current
}

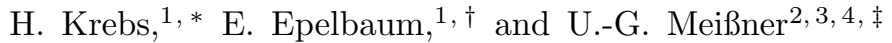 \\ ${ }^{1}$ Ruhr-Universität Bochum, Fakultät für Physik und Astronomie, \\ Institut für Theoretische Physik II, D-44780 Bochum, Germany \\ ${ }^{2}$ Helmholtz-Institut für Strahlen- und Kernphysik and Bethe Center for Theoretical Physics, \\ Universität Bonn, D-53115 Bonn, Germany \\ ${ }^{3}$ Institute for Advanced Simulation, Institut für Kernphysik and Jülich Center for Hadron Physics, \\ Forschungszentrum Jülich, D-52425 Jülich, Germany \\ ${ }^{4}$ Tbilisi State University, 0186 Tbilisi, Georgia
}

(Dated: January 14, 2020)

\begin{abstract}
Recently, we have worked out the axial two-nucleon current operator to leading one-loop order in chiral effective field theory using the method of unitary transformation. Our final expressions, however, differ from the ones derived by the JLab-Pisa group using time-ordered perturbation theory (Phys. Rev. C 93, no. 1, 015501 (2016) Erratum: [Phys. Rev. C 93, no. 4, 049902 (2016)] Erratum: [Phys. Rev. C 95, no. 5, 059901 (2017)]). In this paper we consider the box diagram contribution to the axial current and demonstrate that the results obtained using the two methods are unitary equivalent at the Fock-space level. We adjust the unitary phases by matching the corresponding two-pion exchange nucleon-nucleon potentials and rederive the box diagram contribution to the axial current operator following the approach of the JLab-Pisa group, thereby reproducing our original result. We provide a detailed information on the calculation including the relevant intermediate steps in order to facilitate a clarification of this disagreement.
\end{abstract}

PACS numbers: 13.75.Cs,21.30.-x

\section{INTRODUCTION}

Nuclear axial-vector current operators have been first addressed in the framework of chiral effective field theory (EFT) in Ref. 1. The dominant single-nucleon contribution emerges at order $Q^{-3}$, with $Q$ denoting the expansion parameter of chiral EFT, from the standard Gamow-Teller operator. Contributions to the exchange axial charge and current operators for general kinematical conditions have been recently worked out to order $Q$ by the Bochum-Bonn group [2] using the method of unitary transformation and independently by the JLab-Pisa group [3] using time-ordered perturbation theory. The latter approach relies on the transfer matrix and defines the effective potential and current operators by subtracting the corresponding iterative contributions. Generally, nuclear interactions derived using different methods are expected to be equivalent modulo off-shell effects. However, a direct comparison of the results for e.g. the two-nucleon two-pion-exchange contributions proportional to $g_{A}^{5}$, where $g_{A}$ denotes the nucleon axial coupling constant, given in Eqs. (5.29) and (5.31) of our work [2] and those in Eqs. (7.4), (7.5) of Ref. [3], see also Eqs. (16), (17) of Ref. [4, lets one conclude that both results cannot be unitarily equivalent for the class of unitary transformations considered in [2, thus indicating that at least one of the calculations should be incorrect (unless we have misinterpreted the approach and/or conventions of Refs. [3, 4,). To shed light on this issue and to enable a more direct comparison between the two approaches, we present in this paper a detailed calculation of the contribution of the box diagram e8 in Fig. 4 of 3 to the exchange axial current density, which leads to the already mentioned problematic terms $\propto g_{A}^{5}$. Specifically, we re-derive the corresponding expressions using the method of the JLab-Pisa group, thereby reproducing our original results. To facilitate the error diagnostics and a more detailed comparison, we also provide various intermediate-stage expressions of our calculations.

*Email: hermann.krebs@rub.de

t Email: evgeny.epelbaum@rub.de

拉年ail: meissner@hiskp.uni-bonn.de 
Our paper is organized as follows. Given that nuclear potentials and currents are scheme-dependent quantities, we first need to clarify the relation between the interactions obtained using both methods. To this aim, we focus in section II on the case without external sources and employ the method of the JLab-Pisa group to derive the expressions for the two-pion exchange potential, which turn out to coincide in both approaches. This allows us to unambiguously fix the phases of two unitary transformations on the purely nucleonic subspace of the Fock space that appear at this chiral order. We then give the Fock-space expression for the effective potential of the JLab-Pisa group up to order $Q^{3}$ in terms of the pion-nucleon vertex $\propto g_{A}$ and the corresponding energy denominators, including relativistic corrections that are not related to the expansion of the $g_{A}$-vertex. Having fixed the unitary phases as described above, we use the JLab-Pisa method to derive the Fock-space expressions for the axial-vector operators involving 1, 3 and 5 pion-nucleon vertices $\propto g_{A}$. The resulting expressions are verified to be unitarily equivalent to the ones obtained in Ref. [2] using the method of unitary transformation. Next, in section III] we use the derived Fock-space operators to calculate the non-pion-pole contributions of the box and crossed-box diagrams to the axial-vector current. We give expressions for the current before evaluating the loop integrals, perform the Passarino-Veltman reduction of the relevant tensor integrals and provide explicit expressions for the remaining scalar integrals. The results of this paper are briefly summarized in section IV.

\section{NUCLEAR FORCES UP TO NLO: FIXING THE UNITARY AMBIGUITY}

To derive the effective nuclear potential we start with Eqs. (12)-(15) of Ref. [5]. The half-off-shell transfer matrix $T^{(n)}$ and the free Green's function $G_{0}$ in these equations depend on the energy $E_{i}$ of the initial state (see Eq. (8) of [5]). Since we focus here on the box diagrams, we only retain the leading pion-nucleon vertex $\propto g_{A}$, and we also do not consider the relativistic corrections to this vertex. The inversion of the half-off-shell transfer matrix via Eqs. (12)-(15) of [5] leads to the effective potential, that is identical to the one obtained using the so-called folded-diagram technique [6], see also Ref. []:

$$
\begin{aligned}
v_{\mathrm{FD}}^{(0)} & =-\eta V \frac{\lambda^{1}}{\omega} V \eta, \\
v_{\mathrm{FD}}^{(1)} & =\eta V \mathcal{E} \frac{\lambda^{1}}{\omega^{2}} V \eta-\eta V \frac{\lambda^{1}}{\omega^{2}} V \eta H_{0} \eta \\
v_{\mathrm{FD}}^{(2)} & =2 \eta V \mathcal{E} \frac{\lambda^{1}}{\omega^{3}} V \eta H_{0} \eta-\eta V \frac{\lambda^{1}}{\omega^{3}} V \eta H_{0}^{2} \eta-\eta V \mathcal{E}^{2} \frac{\lambda^{1}}{\omega^{3}} V \eta+\eta V \frac{\lambda^{1}}{\omega^{2}} V \eta V \frac{\lambda^{1}}{\omega} V \eta-\eta V \frac{\lambda^{1}}{\omega} V \frac{\lambda^{2}}{\omega} V \frac{\lambda^{1}}{\omega} V \eta \\
v_{\mathrm{FD}}^{(3)} & =\eta V \frac{\lambda^{1}}{\omega^{3}} V \eta H_{0} \eta V \frac{\lambda^{1}}{\omega} V \eta+\eta V \frac{\lambda^{1}}{\omega^{3}} V \eta V \frac{\lambda^{1}}{\omega} V \eta H_{0} \eta+\eta V \frac{\lambda^{1}}{\omega^{2}} V \eta V \frac{\lambda^{1}}{\omega^{2}} V \eta H_{0} \eta-\eta V \frac{\lambda^{1}}{\omega^{2}} V \frac{\lambda^{2}}{\omega} V \frac{\lambda^{1}}{\omega} V \eta H_{0} \eta \\
& -\eta V \frac{\lambda^{1}}{\omega} V \frac{\lambda^{2}}{\omega^{2}} V \frac{\lambda^{1}}{\omega} V \eta H_{0} \eta-\eta V \frac{\lambda^{1}}{\omega} V \frac{\lambda^{2}}{\omega} V \frac{\lambda^{1}}{\omega^{2}} V \eta H_{0} \eta-2 \eta V \mathcal{E} \frac{\lambda^{1}}{\omega^{3}} V \eta V \frac{\lambda^{1}}{\omega} V \eta+\eta V \mathcal{E} \frac{\lambda^{1}}{\omega^{2}} V \frac{\lambda^{2}}{\omega} V \frac{\lambda^{1}}{\omega} V \eta \\
& -\eta V \frac{\lambda^{1}}{\omega^{2}} V \eta V \mathcal{E} \frac{\lambda^{1}}{\omega^{2}} V \eta+\eta V \frac{\lambda^{1}}{\omega} V \mathcal{E} \frac{\lambda^{2}}{\omega^{2}} V \frac{\lambda^{1}}{\omega} V \eta+\eta V \frac{\lambda^{1}}{\omega} V \frac{\lambda^{2}}{\omega} V \mathcal{E} \frac{\lambda^{1}}{\omega^{2}} V \eta \cdot
\end{aligned}
$$

Here and in what follows, $\eta\left(\lambda^{i}\right)$ denotes the projection operator onto the purely nucleonic states of the Fock space (states involving $i$ pions), $V$ is the operator corresponding to the pion-nucleon vertex $\propto g_{A}{ }^{1}$, while $\omega$ and $\mathcal{E}$ denote the sum of $n$ pion energies $\omega_{i}=\sqrt{\vec{p}_{i}^{2}+M_{\pi}^{2}}$ and the kinetic energy of nucleons in an intermediate state $\lambda^{n}$, respectively. Further, the superscript $(n)$ gives the order $Q$ of the chiral expansion. Following the approach of Refs. [3 5 , we count the nucleon mass as $m_{N} \sim \Lambda_{\chi}$, with $\Lambda_{\chi}$ denoting the breakdown scale of chiral EFT. The calculations by the Bochum-Bonn group employ the counting scheme with $Q / m_{N} \sim\left(Q / \Lambda_{\chi}\right)^{2}$.

The manifestly non-hermitean effective potential in Eq. (1) is uniquely determined for a given half-off-shell T-matrix. Changing the off-shell behaviour of the T-matrix by adding terms proportional to $\left[H_{0}, X\right]$, where $X$ is an arbitrary operator, and applying the same inversion procedure leads to a different effective potential. Off-shell changes of the T-matrix can be understood in terms of similarity transformations of the effective Hamiltonian. Since the authors

\footnotetext{
1 In our earlier paper [2], we used for this vertex the notation $H_{2,1}^{(1)}$ in order to signify that it involves two nucleon fields and one pion field and has the dimension $\kappa=1$ as defined in Ref. [2]. Since we only consider this type of vertex here, we choose to employ a simpler notation.
} 
of Ref. [5] do not specify the operator $X$, we need to extract the off-shell behavior of the T-matrix from their final expressions for the effective potential. To derive nuclear forces in the convention of the JLab-Pisa group, we apply a series of similarity transformations on the potential $v_{\mathrm{FD}}$ of Eq. (1). We first bring $v_{\mathrm{FD}}$ into a hermitean form by applying a similarity transformation [7, 8]

$$
v_{\text {Okubo }}=\left(1+A A^{\dagger}+A^{\dagger} A\right)^{1 / 2} v_{\mathrm{FD}}\left(1+A A^{\dagger}+A^{\dagger} A\right)^{-1 / 2} .
$$

Here, $v_{\text {Okubo }}$ is precisely the hermitean potential that is obtained from the underlying pion-nucleon Hamiltonian $H$ via the unitary transformation introduced by Okubo [9],

$$
v_{\text {Okubo }}=U^{\dagger} H U-H_{0},, \quad U=\left(\begin{array}{cc}
\left(\eta+A^{\dagger} A\right)^{-1 / 2} & -A^{\dagger}\left(1+A A^{\dagger}\right)^{-1 / 2} \\
A\left(1+A^{\dagger} A\right)^{-1 / 2} & \left(\lambda+A A^{\dagger}\right)^{-1 / 2}
\end{array}\right),
$$

with the operator $A=\lambda A \eta$ satisfying the nonlinear decoupling equation

$$
\lambda(H-[A, H]-A H A) \eta=0
$$

Notice that the Okubo unitary transformation leads to non-factorizable and non-renormalizable nuclear potentials [10]. For the class of contributions considered in this work, renormalizability of the nuclear potentials can be restored by performing additional $\eta$-space unitary transformations

$$
v=U_{12}^{\dagger}\left(v_{\text {Okubo }}+H_{0}\right) U_{12}-H_{0}
$$

with

$$
U_{12}=\exp \left(\alpha_{1} S_{1}+\alpha_{2} S_{2}\right)
$$

where the antihermitean operators $S_{1}$ and $S_{2}$ are defined in Eq. (3.25) of [10]. To fix the unitary phases $\alpha_{1}$ and $\alpha_{2}$ we match the expression for the two-pion exchange two-nucleon potential obtained from $v$ with Eq. (19) of Ref. [5]. We reproduce the expression in Eq. (19) of Ref. [5] provided the unitary phases $\alpha_{1}$ and $\alpha_{2}$ are chosen to be

$$
\alpha_{1}=-\frac{1}{2}, \quad \alpha_{2}=\frac{1}{4} .
$$

This particular choice leads to renormalizable nuclear potentials [10] and is employed also by the Bochum-Bonn group.

The leading relativistic corrections to the nuclear forces are well known to depend on two arbitrary phases $\bar{\beta}_{8}, \bar{\beta}_{9}$, see Eq. (1.4) of Ref. [1] for the definition. In Ref. [12, the same off-shell ambiguity is expressed in terms of the phases $\mu, \nu$. To be consistent with the choice made by the JLab-Pisa group for the one-pion exchange potential, see Eq. (19) of Ref. [5], we set $\nu=0$ which corresponds to $\beta_{8}=\nu / 2=0$ in our notation. The second phase needs not be discussed here since we do not consider relativistic corrections to the $g_{A}$-vertex.

With the above choices, we arrive at the Fock-space expressions for the effective potential that correspond to the convention of the JLab-Pisa group:

$$
\begin{aligned}
v^{(0)} & =-\eta V \frac{\lambda^{1}}{\omega} V \eta \\
v^{(1)} & =\frac{1}{2} \eta V \mathcal{E} \frac{\lambda^{1}}{\omega^{2}} V \eta-\frac{1}{2} \eta H_{0} \eta V \frac{\lambda^{1}}{\omega^{2}} V \eta+\text { h.c. } \\
v^{(2)} & =\eta H_{0} \eta V \mathcal{E} \frac{\lambda^{1}}{\omega^{3}} V \eta-\frac{1}{2} \eta H_{0}^{2} \eta V \frac{\lambda^{1}}{\omega^{3}} V \eta-\frac{1}{2} \eta V \mathcal{E}^{2} \frac{\lambda^{1}}{\omega^{3}} V \eta+\frac{1}{2} \eta V \frac{\lambda^{1}}{\omega^{2}} V \eta V \frac{\lambda^{1}}{\omega} V \eta-\frac{1}{2} \eta V \frac{\lambda^{1}}{\omega} V \frac{\lambda^{2}}{\omega} V \frac{\lambda^{1}}{\omega} V \eta \\
& + \text { h.c. }, \\
v^{(3)} & =\alpha_{1}\left(\eta H_{0} \eta V \frac{\lambda^{1}}{\omega} V \eta V \frac{\lambda^{1}}{\omega^{3}} V \eta-\eta H_{0} \eta V \frac{\lambda^{1}}{\omega^{3}} V \eta V \frac{\lambda^{1}}{\omega} V \eta\right)
\end{aligned}
$$

\footnotetext{
2 Note that there is a misprint in Eq. (3.31) of [10], a factor of 2 in front of $\alpha_{2}$ is missing. The corrected equation reads $\alpha_{1}=-2 \alpha_{2}=-\frac{1}{2}$.
} 


$$
\begin{aligned}
& +\alpha_{2}\left(\eta H_{0} \eta V \frac{\lambda^{1}}{\omega} V \frac{\lambda^{2}}{\omega} V \frac{\lambda^{1}}{\omega^{2}} V \eta-\eta H_{0} \eta V \frac{\lambda^{1}}{\omega^{2}} V \frac{\lambda^{2}}{\omega} V \frac{\lambda^{1}}{\omega} V \eta\right) \\
& +\frac{3}{8} \eta H_{0} \eta V \frac{\lambda^{1}}{\omega^{2}} V \eta V \frac{\lambda^{1}}{\omega^{2}} V \eta-\frac{1}{2} \eta H_{0} \eta V \frac{\lambda^{1}}{\omega^{2}} V \frac{\lambda^{2}}{\omega} V \frac{\lambda^{1}}{\omega} V \eta+\frac{1}{2} \eta H_{0} \eta V \frac{\lambda^{1}}{\omega} V \eta V \frac{\lambda^{1}}{\omega^{3}} V \eta-\frac{1}{2} \eta H_{0} \eta V \frac{\lambda^{1}}{\omega} V \frac{\lambda^{2}}{\omega^{2}} V \frac{\lambda^{1}}{\omega} V \eta \\
& -\frac{1}{2} \eta H_{0} \eta V \frac{\lambda^{1}}{\omega} V \frac{\lambda^{2}}{\omega} V \frac{\lambda^{1}}{\omega^{2}} V \eta+\frac{1}{2} \eta V \frac{\lambda^{1}}{\omega^{3}} V \eta H_{0} \eta V \frac{\lambda^{1}}{\omega} V \eta+\frac{1}{8} \eta V \frac{\lambda^{1}}{\omega^{2}} V \eta H_{0} \eta V \frac{\lambda^{1}}{\omega^{2}} V \eta-\eta V \mathcal{E} \frac{\lambda^{1}}{\omega^{3}} V \eta V \frac{\lambda^{1}}{\omega} V \eta \\
& -\frac{1}{2} \eta V \mathcal{E} \frac{\lambda^{1}}{\omega^{2}} V \eta V \frac{\lambda^{1}}{\omega^{2}} V \eta+\eta V \mathcal{E} \frac{\lambda^{1}}{\omega^{2}} V \frac{\lambda^{2}}{\omega} V \frac{\lambda^{1}}{\omega} V \eta+\frac{1}{2} \eta V \frac{\lambda^{1}}{\omega} V \mathcal{E} \frac{\lambda^{2}}{\omega^{2}} V \frac{\lambda^{1}}{\omega} V \eta+\text { h.c. }
\end{aligned}
$$

We have retained here the dependence on the phases $\alpha_{1,2}$ which should be chosen according to Eq. (7). A comparison of Eqs. (8)-(11) with Eq. (3.13) of [13 $]^{3}$, obtained using the Okubo transformation, shows that both results indeed coincide for the case of $\alpha_{1}=\alpha_{2}=0$ as already pointed out above. We further emphasize that we have neglected all relativistic corrections in Eqs. (9), (10) and (11) which scale as $1 / m_{N}^{n}$ with $n>2(n>1)$ for operators involving two (four) insertions of the pion-nucleon vertex $V$. The neglected effects are of a higher order in the chiral expansion.

We now turn to the axial-vector currents. As in the case of the nuclear forces, effective current operators can be derived by inverting the contribution to the T-matrix $T_{5}$ that depends linearly on the corresponding external sources. This in turn is the approach followed by the JLab-Pisa group to derive the axial-vector current operator $v_{5}$, see Eqs. (3.8)-(3.12) of [3]. In these equations the operators $T_{5}$ and $G_{0}$ depend on the initial- and final-state energies $E_{i}$ and $E_{f}$. The relation between the axial-vector current $v_{5}\left(E_{f}-E_{i}\right)$ and the T-matrix $T_{5}$ is derived in Appendix A and given by

$$
T_{5}\left(E_{f}, E_{i}\right)=\left(1-v G_{0}\left(E_{f}\right)\right)^{-1} v_{5}\left(E_{f}-E_{i}\right)\left(1-G_{0}\left(E_{i}\right) v\right)^{-1} .
$$

Here, we have already exploited the fact that the axial-vector source appears in first order perturbation theory. The explicit dependence of the interaction on the axial-vector source is hidden in $v_{5}\left(E_{f}-E_{i}\right)$. Its dependence on the energy difference $E_{f}-E_{i}$ emerges due to the explicit time-dependence of axial-vector source.

We now invert Eq. 12 in the same way as done by the JLab-Pisa group ${ }^{4}$ to obtain the axial-vector current operator using Eqs. 8), (9), (10) and (11) for the strong-interaction potential $v$. The resulting somewhat lengthy Fock-space expressions are given in Appendix B Notice that exactly the same expressions are obtained using the method of unitary transformation in Ref. 2, provided all additional unitary transformations that depend explicitly on the external axial-vector sources are switched off, i.e. $\alpha_{i}^{a x}=0$ for all $i$. This demonstrates that the current operators derived by the two groups should be unitarily equivalent.

\section{CALCULATION OF THE BOX DIAGRAM}

We now use the expressions for $v_{5}$ given in Appendix $\mathrm{B}$ to calculate the contributions of the box diagrams to the axial-vector current operator, which are visualized in Fig. 1. While we focus here exclusively on the non-pion-pole contributions, the Fock-space expressions for the pion-pole terms are also provided in Appendix B. Each diagram shown in Fig. 1 gives rise to a series of time-ordered graphs, whose contributions can be obtained by calculating the two-nucleon matrix elements of the operators in Eq. (B.6). Time-ordered graphs associated with any of the six diagrams in Fig. 1 feature the same sequence of vertices and thus have the same spin-isospin-momentum structure which will be given below. We begin with collecting together the energy denominators for diagrams (1)-(6) and obtain the following result:

$$
\operatorname{box}(1)=\frac{2}{\omega_{l} \omega_{\vec{l}+\vec{q}_{2}}^{3}}+\left(\alpha_{1}+\frac{1}{2}\right)\left[\frac{4}{\omega_{l}^{3} \omega_{\vec{l}+\vec{q}_{2}}}-\frac{4}{\omega_{l} \omega_{\vec{l}+\vec{q}_{2}}^{3}}\right]
$$

\footnotetext{
${ }^{3}$ In Eq. (3.13) of [13], the author discusses the Yukawa model and not chiral effective field theory. This is, however, perfectly sufficient for our purpose since we are only interested in the box diagram contributions. The only vertex which is relevant for the current discussion of effective potentials is the leading one-pion-nucleon vertex $V$ which can be interpreted as a Yukawa-type interaction.

4 The explicit energy dependence as given in Eq. $\sqrt{12}$ is not spelled out in Eqs. (3.8)-(3.12) of [3].
} 


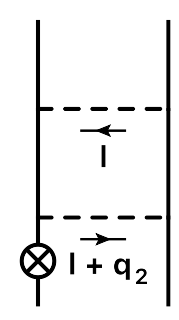

(1)

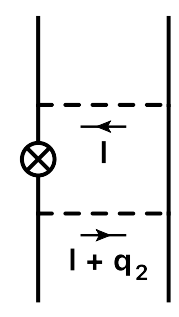

(2)

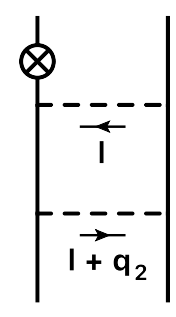

(3)

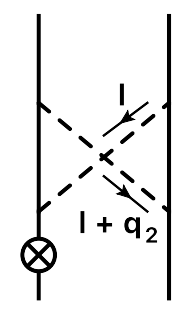

(4)

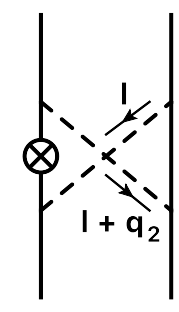

(5)

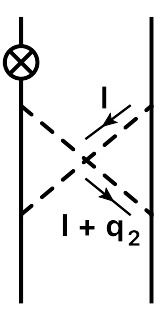

(6)

FIG. 1: Box diagrams proportional to $g_{A}^{5}$. Diagrams obtained by permutation of the nucleon labels $1 \leftrightarrow 2$ are not shown. Crossed circles denote the coupling of axial-vector source $\propto g_{A}$. The momenta $\vec{l}$ and $\vec{l}+\vec{q}_{2}$ in the static pion propagators are explicitly spelled out. Initial (final) momenta momenta of the nucleons are denoted by $\vec{p}_{1}$ and $\vec{p}_{2}\left(\vec{p}_{1}^{\prime}\right.$ and $\left.\vec{p}_{2}^{\prime}\right)$. The momentum transfer of the nucleon $j$ is defined by $\vec{q}_{j}=\vec{p}_{j}^{\prime}-\vec{p}_{J}$ with $j=1,2$.

$$
+\left(\alpha_{2}-\frac{1}{4}\right)\left[\frac{2}{\omega_{l}^{2} \omega_{\vec{l}+\vec{q}_{2}}\left(\omega_{\vec{l}+\vec{q}_{2}}+\omega_{l}\right)}-\frac{2}{\omega_{l} \omega_{\vec{l}+\vec{q}_{2}}^{2}\left(\omega_{\vec{l}+\vec{q}_{2}}+\omega_{l}\right)}\right],
$$

$\operatorname{box}(2)=-\frac{2}{\omega_{l} \omega_{\vec{l}+\vec{q}_{2}}^{3}}-\frac{2}{\omega_{l}^{3} \omega_{\vec{l}+\vec{q}_{2}}}$,

$\operatorname{box}(3)=\frac{2}{\omega_{l}^{3} \omega_{\vec{l}+\vec{q}_{2}}}+\left(\alpha_{1}+\frac{1}{2}\right)\left[\frac{4}{\omega_{l} \omega_{\vec{l}+\vec{q}_{2}}^{3}}-\frac{4}{\omega_{l}^{3} \omega_{\vec{l}+\vec{q}_{2}}}\right]$

$+\left(\alpha_{2}-\frac{1}{4}\right)\left[\frac{2}{\omega_{l} \omega_{\vec{l}+\vec{q}_{2}}^{2}\left(\omega_{\vec{l}+\vec{q}_{2}}+\omega_{l}\right)}-\frac{2}{\omega_{l}^{2} \omega_{\vec{l}+\vec{q}_{2}}\left(\omega_{\vec{l}+\vec{q}_{2}}+\omega_{l}\right)}\right]$,

$\operatorname{box}(4)=-\frac{2}{\omega_{l}^{3} \omega_{\vec{l}+\vec{q}_{2}}}$,

$\operatorname{box}(5)=\frac{2}{\omega_{l} \omega_{\vec{l}+\vec{q}_{2}}^{3}}+\frac{2}{\omega_{l}^{3} \omega_{\vec{l}+\vec{q}_{2}}}$,

$\operatorname{box}(6)=-\frac{2}{\omega_{l} \omega_{\vec{l}+\vec{q}_{2}}^{3}}$.

For the choice of the unitary phases $\alpha_{1}$ and $\alpha_{2}$ made by our and by the JLab-Pisa group given in Eq. (7), all terms in the square brackets vanish leading to the factorized results for every diagrams.

Combining the energy denominators in Eq. (13) with the corresponding spin-isospin-momentum structures, the complete contribution of the box diagrams to the axial-vector current can be written in the form

$$
\overrightarrow{\mathcal{A}}_{\mathrm{box}}^{a}=\sum_{i=1}^{6} \int \frac{d^{3} l}{(2 \pi)^{3}} \overrightarrow{\mathcal{A}}_{\mathrm{box}(i)}^{a}+(1 \leftrightarrow 2),
$$

where $a$ is an isospin index and the $\overrightarrow{\mathcal{A}}_{\text {box }(i)}$ are given by

$$
\begin{aligned}
\overrightarrow{\mathcal{A}}_{\text {box }(1)}^{a} & =\frac{g_{A}^{5}}{64 F_{\pi}^{4} \omega_{l}^{2} \omega_{\vec{l}+\vec{q}_{2}}^{4}}\left(2 i\left[\tau_{1} \times \tau_{2}\right]^{a}-3 \tau_{1}^{a}+2 \tau_{2}^{a}\right)\left(\vec { l } \left[-i \vec{q}_{2} \cdot \vec{\sigma}_{1} \vec{l} \cdot \vec{q}_{2} \times \vec{\sigma}_{2}-\left(l^{2}+\vec{l} \cdot \vec{q}_{2}\right) \vec{q}_{2} \cdot \vec{\sigma}_{1}\right.\right. \\
& \left.+q_{2}^{2} \vec{l} \cdot \vec{\sigma}_{2}-\vec{l} \cdot \vec{q}_{2} \vec{q}_{2} \cdot \vec{\sigma}_{2}\right]+\vec{q}_{2}\left[i \vec{l} \cdot \vec{\sigma}_{1} \vec{l} \cdot \vec{q}_{2} \times \vec{\sigma}_{2}+\vec{l} \cdot \vec{q}_{2}\left(\vec{l} \cdot \vec{\sigma}_{1}-\vec{l} \cdot \vec{\sigma}_{2}\right)+l^{2}\left(\vec{l} \cdot \vec{\sigma}_{1}+\vec{q}_{2} \cdot \vec{\sigma}_{2}\right)\right] \\
& \left.+\vec{\sigma}_{1}\left(l^{2}+\vec{l} \cdot \vec{q}_{2}\right)\left(i \vec{l} \cdot \vec{q}_{2} \times \vec{\sigma}_{2}+l^{2}+\vec{l} \cdot \vec{q}_{2}\right)+\vec{\sigma}_{2}\left[\left(\vec{l} \cdot \vec{q}_{2}\right)^{2}-l^{2} q_{2}^{2}\right]+i \vec{l} \times \vec{q}_{2}\left(l^{2}+\vec{l} \cdot \vec{q}_{2}\right)\right), \\
\overrightarrow{\mathcal{A}}_{\text {box }(2)}^{a} & =\frac{g_{A}^{5}\left(\omega_{\vec{l}+\vec{q}_{2}}^{2}+\omega_{l}^{2}\right)}{64 F_{\pi}^{4} \omega_{l}^{4} \omega_{\vec{l}+\vec{q}_{2}}^{4}}\left(2 \tau_{2}^{a}-\tau_{1}^{a}\right)\left(-\vec{l}\left[-i \vec{l} \cdot \vec{\sigma}_{1} \vec{l} \cdot \vec{q}_{2} \times \vec{\sigma}_{2}-i \vec{q}_{2} \cdot \vec{\sigma}_{1} \vec{l} \cdot \vec{q}_{2} \times \vec{\sigma}_{2}-\vec{l} \cdot \overrightarrow{q_{2}}\left(2 \vec{l} \cdot \vec{\sigma}_{1}\right.\right.\right.
\end{aligned}
$$




$$
\begin{aligned}
& \left.\left.+\vec{q}_{2} \cdot \vec{\sigma}_{1}+\vec{q}_{2} \cdot \vec{\sigma}_{2}\right)-l^{2}\left(2 \vec{l} \cdot \vec{\sigma}_{1}+\vec{q}_{2} \cdot \vec{\sigma}_{1}\right)+q_{2}^{2} \vec{l} \cdot \vec{\sigma}_{2}\right]-i \vec{l} \times \vec{q}_{2}\left[-\left(\vec{l} \cdot \vec{\sigma}_{1}\right)\left(\vec{l} \cdot \vec{\sigma}_{2}+\vec{q}_{2} \cdot \vec{\sigma}_{2}\right)\right. \\
& \left.+\left(l^{2}+\vec{l} \cdot \vec{q}_{2}\right)\left(\vec{\sigma}_{1} \cdot \vec{\sigma}_{2}+1\right)\right]-i \vec{l} \times \vec{\sigma}_{2}\left[q_{2}^{2} \vec{l} \cdot \vec{\sigma}_{1}+\vec{l} \cdot \vec{q}_{2}\left(\vec{l} \cdot \vec{\sigma}_{1}-\vec{q}_{2} \cdot \vec{\sigma}_{1}\right)-l^{2} \vec{q}_{2} \cdot \vec{\sigma}_{1}\right] \\
& \left.-\vec{q}_{2}\left[l^{2}\left(\vec{q}_{2} \cdot \vec{\sigma}_{2}-\vec{l} \cdot \vec{\sigma}_{1}\right)-\vec{l} \cdot \vec{q}_{2}\left(\vec{l} \cdot \vec{\sigma}_{1}+\vec{l} \cdot \vec{\sigma}_{2}\right)\right]-\vec{\sigma}_{1}\left(l^{2}+\vec{l} \cdot \vec{q}_{2}\right)^{2}-\vec{\sigma}_{2}\left[\left(\vec{l} \cdot \vec{q}_{2}\right)^{2}-l^{2} q_{2}^{2}\right]\right), \\
& \overrightarrow{\mathcal{A}}_{\text {box (3) }}^{a}=\frac{g_{A}^{5}}{64 F_{\pi}^{4} \omega_{l}^{4} \omega_{\vec{l}+\vec{q}_{2}}^{2}}\left(2 i\left[\tau_{1} \times \tau_{2}\right]^{a}+3 \tau_{1}^{a}-2 \tau_{2}^{a}\right)\left(-\vec{l}\left[i\left(\vec{l} \cdot \vec{\sigma}_{1}+\vec{q}_{2} \cdot \vec{\sigma}_{1}\right) \vec{l} \cdot \vec{q}_{2} \times \vec{\sigma}_{2}+\left(l^{2}+\vec{l} \cdot \vec{q}_{2}\right) \vec{q}_{2} \cdot \vec{\sigma}_{1}\right.\right. \\
& \left.+q_{2}^{2} \vec{l} \cdot \vec{\sigma}_{2}-\vec{l} \cdot \vec{q}_{2} \vec{q}_{2} \cdot \vec{\sigma}_{2}\right]-i \vec{l} \times \vec{q}_{2}\left[-\vec{l} \cdot \vec{\sigma}_{1}\left(\vec{l} \cdot \vec{\sigma}_{2}+\vec{q}_{2} \cdot \vec{\sigma}_{2}\right)+\left(l^{2}+\vec{l} \cdot \vec{q}_{2}\right)\left(\vec{\sigma}_{1} \cdot \vec{\sigma}_{2}+1\right)\right] \\
& -i \vec{l} \times \vec{\sigma}_{2}\left[\left(\vec{l} \cdot \vec{q}_{2}+q_{2}^{2}\right) \vec{l} \cdot \vec{\sigma}_{1}-\left(l^{2}+\vec{l} \cdot \vec{q}_{2}\right) \vec{q}_{2} \cdot \vec{\sigma}_{1}\right]-\vec{q}_{2}\left[l^{2}\left(\vec{q}_{2} \cdot \vec{\sigma}_{2}-\vec{l} \cdot \vec{\sigma}_{1}\right)-\vec{l} \cdot \vec{q}_{2}\left(\vec{l} \cdot \vec{\sigma}_{1}+\vec{l} \cdot \vec{\sigma}_{2}\right)\right] \\
& \left.-\vec{\sigma}_{1}\left(l^{2}+\vec{l} \cdot \vec{q}_{2}\right)^{2}-\vec{\sigma}_{2}\left[\left(\vec{l} \cdot \vec{q}_{2}\right)^{2}-l^{2} q_{2}^{2}\right]\right) \text {, } \\
& \overrightarrow{\mathcal{A}}_{\text {box (4) }}^{a}=\frac{g_{A}^{5}}{64 F_{\pi}^{4} \omega_{l}^{4} \omega_{\vec{l}+\vec{q}_{2}}^{2}}\left(2 i\left[\tau_{1} \times \tau_{2}\right]^{a}+3 \tau_{1}^{a}+2 \tau_{2}^{a}\right)\left(\vec { l } \left[i \vec{q}_{2} \cdot \vec{\sigma}_{1} \vec{l} \cdot \vec{q}_{2} \times \vec{\sigma}_{2}+\left(l^{2}+\vec{l} \cdot \vec{q}_{2}\right) \vec{q}_{2} \cdot \vec{\sigma}_{1}\right.\right. \\
& \left.-q_{2}^{2} \vec{l} \cdot \vec{\sigma}_{2}+\vec{l} \cdot \vec{q}_{2} \vec{q}_{2} \cdot \vec{\sigma}_{2}\right]+\vec{q}_{2}\left[-i \vec{l} \cdot \vec{\sigma}_{1} \vec{l} \cdot \vec{q}_{2} \times \vec{\sigma}_{2}+\vec{l} \cdot \vec{q}_{2}\left(\vec{l} \cdot \vec{\sigma}_{2}-\vec{l} \cdot \vec{\sigma}_{1}\right)-l^{2}\left(\vec{l} \cdot \vec{\sigma}_{1}+\vec{q}_{2} \cdot \vec{\sigma}_{2}\right)\right] \\
& \left.+\vec{\sigma}_{1}\left(l^{2}+\vec{l} \cdot \vec{q}_{2}\right)\left(i \vec{l} \cdot \vec{q}_{2} \times \vec{\sigma}_{2}+\vec{l} \cdot \vec{q}_{2}+l^{2}\right)+\vec{\sigma}_{2}\left[l^{2} q_{2}^{2}-\left(\vec{l} \cdot \vec{q}_{2}\right)^{2}\right]-i \vec{l} \times \vec{q}_{2}\left[\vec{l} \cdot \vec{q}_{2}+l^{2}\right]\right), \\
& \overrightarrow{\mathcal{A}}_{\text {box }(5)}^{a}=\frac{g_{A}^{5}\left(\omega_{\vec{l}+\vec{q}_{2}}^{2}+\omega_{l}^{2}\right)}{64 F_{\pi}^{4} \omega_{l}^{4} \omega_{\vec{l}+\vec{q}_{2}}^{4}}\left(2 \tau_{2}^{a}+\tau_{1}^{a}\right)\left(\vec { l } \left[i \vec{l} \cdot \vec{\sigma}_{1} \vec{l} \cdot \vec{q}_{2} \times \vec{\sigma}_{2}+\vec{l} \cdot \vec{q}_{2}\left(2 \vec{l} \cdot \vec{\sigma}_{1}+\vec{q}_{2} \cdot \vec{\sigma}_{1}-\vec{q}_{2} \cdot \vec{\sigma}_{2}\right)\right.\right. \\
& \left.+l^{2}\left(2 \vec{l} \cdot \vec{\sigma}_{1}+\vec{q}_{2} \cdot \vec{\sigma}_{1}\right)+q_{2}^{2} \vec{l} \cdot \vec{\sigma}_{2}\right]+\vec{q}_{2}\left[i \vec{l} \cdot \vec{\sigma}_{1} \vec{l} \cdot \vec{q}_{2} \times \vec{\sigma}_{2}+\vec{l} \cdot \vec{q}_{2}\left(\vec{l} \cdot \vec{\sigma}_{1}-\vec{l} \cdot \vec{\sigma}_{2}\right)+l^{2}\left(\vec{l} \cdot \vec{\sigma}_{1}+\vec{q}_{2} \cdot \vec{\sigma}_{2}\right)\right] \\
& -i \vec{l} \times \vec{q}_{2}\left[-\vec{l} \cdot \vec{\sigma}_{2}\left(\vec{l} \cdot \vec{\sigma}_{1}+\vec{q}_{2} \cdot \vec{\sigma}_{1}\right)+\left(l^{2}+\vec{l} \cdot \vec{q}_{2}\right)\left(\vec{\sigma}_{1} \cdot \vec{\sigma}_{2}-1\right)\right]+i \vec{l} \times \vec{\sigma}_{2}\left[l^{2} \vec{q}_{2} \cdot \vec{\sigma}_{1}-\vec{l} \cdot \overrightarrow{q_{2}} \vec{l} \cdot \vec{\sigma}_{1}\right] \\
& \left.+i \vec{q}_{2} \times \vec{\sigma}_{2}\left[l^{2} \vec{q}_{2} \cdot \vec{\sigma}_{1}-\vec{l} \cdot \overrightarrow{q_{2}} \vec{l} \cdot \vec{\sigma}_{1}\right]-\vec{\sigma}_{1}\left(l^{2}+\vec{l} \cdot \vec{q}_{2}\right)^{2}+\vec{\sigma}_{2}\left[\left(\vec{l} \cdot \vec{q}_{2}\right)^{2}-l^{2} q_{2}^{2}\right]\right), \\
& \overrightarrow{\mathcal{A}}_{\text {box (6) }}^{a}=\frac{g_{A}^{5}}{64 F_{\pi}^{4} \omega_{l}^{2} \omega_{\vec{l}+\vec{q}_{2}}^{4}}\left(2 i\left[\tau_{1} \times \tau_{2}\right]^{a}-3 \tau_{1}^{a}-2 \tau_{2}^{a}\right)\left(\vec { l } \left[-i \vec{l} \cdot \vec{\sigma}_{1} \vec{l} \cdot \vec{q}_{2} \times \vec{\sigma}_{2}+\vec{l} \cdot \vec{q}_{2}\left(\vec{q}_{2} \cdot \vec{\sigma}_{1}-\vec{q}_{2} \cdot \vec{\sigma}_{2}\right)\right.\right. \\
& \left.+l^{2} \vec{q}_{2} \cdot \vec{\sigma}_{1}+q_{2}^{2} \vec{l} \cdot \vec{\sigma}_{2}\right]-\vec{q}_{2}\left[i \vec{l} \cdot \vec{\sigma}_{1} \vec{l} \cdot \vec{q}_{2} \times \vec{\sigma}_{2}+\vec{l} \cdot \vec{q}_{2}\left(\vec{l} \cdot \vec{\sigma}_{1}+\vec{l} \cdot \vec{\sigma}_{2}\right)+l^{2}\left(\vec{l} \cdot \vec{\sigma}_{1}-\vec{q}_{2} \cdot \vec{\sigma}_{2}\right)\right] \\
& -i \vec{l} \times \vec{q}_{2}\left[-\vec{l} \cdot \vec{\sigma}_{2}\left(\vec{l} \cdot \vec{\sigma}_{1}+\vec{q}_{2} \cdot \vec{\sigma}_{1}\right)+\left(l^{2}+\vec{l} \cdot \vec{q}_{2}\right)\left(\vec{\sigma}_{1} \cdot \vec{\sigma}_{2}-1\right)\right]-i \vec{l} \times \vec{\sigma}_{2}\left[\vec{l} \cdot \vec{q}_{2} \vec{l} \cdot \vec{\sigma}_{1}-l^{2} \vec{q}_{2} \cdot \vec{\sigma}_{1}\right] \\
& \left.-i \vec{q}_{2} \times \vec{\sigma}_{2}\left[\vec{l} \cdot \overrightarrow{q_{2}} \vec{l} \cdot \vec{\sigma}_{1}-l^{2} \vec{q}_{2} \cdot \vec{\sigma}_{1}\right]-\vec{\sigma}_{1}\left(l^{2}+\vec{l} \cdot \vec{q}_{2}\right)^{2}+\vec{\sigma}_{2}\left[\left(\vec{l} \cdot \vec{q}_{2}\right)^{2}-l^{2} q_{2}^{2}\right]\right) .
\end{aligned}
$$

Here, $F_{\pi}$ denotes the pion decay constant while $\vec{\sigma}_{i}\left(\boldsymbol{\tau}_{i}\right)$ are spin (isospin) Pauli matrices of the nucleon $i$. To further simplify these expressions, we write the scalar products $l^{2} \equiv|\vec{l}|^{2}$ and $\vec{l} \cdot \overrightarrow{q_{2}}$ as linear combinations of the corresponding pion energies via

$$
\begin{aligned}
l^{2} & =\omega_{l}^{2}-M_{\pi}^{2}, \\
\vec{l} \cdot \overrightarrow{q_{2}} & =\frac{1}{2}\left(\omega_{\vec{l}+\vec{q}_{2}}^{2}-\omega_{l}^{2}-q_{2}^{2}\right) .
\end{aligned}
$$

After these simplifications we still have to evaluate tensor integrals up to rank three. To this aim, we carry out the standard Passarino-Veltman reduction in $d$ dimensions:

$$
\begin{aligned}
& \mu^{3-d} \int \frac{d^{d} l}{(2 \pi)^{d}} \frac{l_{i_{1}} l_{i_{2}} l_{i_{3}}}{\omega_{l}^{n_{1}} \omega_{\vec{l} \vec{q}_{2}}^{n_{2}}}=\frac{\left(q_{2}\right)_{i_{3}} \delta_{i_{1}, i_{2}}+\left(q_{2}\right)_{i_{2}} \delta_{i_{1}, i_{3}}+\left(q_{2}\right)_{i_{1}} \delta_{i_{2}, i_{3}}}{8(d-1) q_{2}^{4}}\left(q_{2}^{4}\left(4 M_{\pi}^{2}+q_{2}^{2}\right) s\left(n_{1}, n_{2}\right)\right. \\
- & q_{2}^{2}\left[\left(q_{2}^{2}-4 M_{\pi}^{2}\right) s\left(n_{1}-2, n_{2}\right)+\left(4 M_{\pi}^{2}+3 q_{2}^{2}\right) s\left(n_{1}, n_{2}-2\right)+s\left(n_{1}-4, n_{2}\right)+2 s\left(n_{1}-2, n_{2}-2\right)\right. \\
- & \left.\left.3 s\left(n_{1}, n_{2}-4\right)\right]+s\left(n_{1}-6, n_{2}\right)-3 s\left(n_{1}-4, n_{2}-2\right)+3 s\left(n_{1}-2, n_{2}-4\right)-s\left(n_{1}, n_{2}-6\right)\right) \\
+ & \frac{\left(q_{2}\right)_{i_{1}}\left(q_{2}\right)_{i_{2}}\left(q_{2}\right)_{i_{3}}}{8(d-1) q_{2}^{6}}\left(-q_{2}^{4} s\left(n_{1}, n_{2}\right)\left((d+2) q_{2}^{2}+12 M_{\pi}^{2}\right)-3 d q_{2}^{2} s\left(n_{1}-4, n_{2}\right)\right. \\
+ & 6 d q_{2}^{2} s\left(n_{1}-2, n_{2}-2\right)-3 d q_{2}^{4} s\left(n_{1}-2, n_{2}\right)-3 d q_{2}^{2} s\left(n_{1}, n_{2}-4\right)+3 d q_{2}^{4} s\left(n_{1}, n_{2}-2\right)
\end{aligned}
$$




$$
\begin{aligned}
& -(d+2) s\left(n_{1}-6, n_{2}\right)+3(d+2) s\left(n_{1}-4, n_{2}-2\right)-3 d s\left(n_{1}-2, n_{2}-4\right)+d s\left(n_{1}, n_{2}-6\right) \\
& -\quad 12 M_{\pi}^{2} q_{2}^{2} s\left(n_{1}-2, n_{2}\right)+12 M_{\pi}^{2} q_{2}^{2} s\left(n_{1}, n_{2}-2\right)+6 q_{2}^{2} s\left(n_{1}-4, n_{2}\right)+6 q_{2}^{4} s\left(n_{1}-2, n_{2}\right) \\
& \left.-6 q_{2}^{2} s\left(n_{1}, n_{2}-4\right)+6 q_{2}^{4} s\left(n_{1}, n_{2}-2\right)-6 s\left(n_{1}-2, n_{2}-4\right)+2 s\left(n_{1}, n_{2}-6\right)\right) \\
& \quad \mu^{3-d} \int \frac{d^{d} l}{(2 \pi)^{d}} \frac{l_{i_{1}} l_{i_{2}}}{\omega_{l}^{n_{1}} \omega_{\vec{l}+\vec{q}_{2}}^{n_{2}}}=\frac{\left(q_{2}\right)_{i_{1}}\left(q_{2}\right) i_{2}}{4(d-1) q_{2}^{4}}\left(q_{2}^{2} s\left(n_{1}, n_{2}\right)\left(d q_{2}^{2}+4 M_{\pi}^{2}\right)+d\left[2 q _ { 2 } ^ { 2 } \left(s\left(n_{1}-2, n_{2}\right)\right.\right.\right. \\
& \left.\left.\left.-\quad s\left(n_{1}, n_{2}-2\right)\right)+s\left(n_{1}-4, n_{2}\right)-2 s\left(n_{1}-2, n_{2}-2\right)+s\left(n_{1}, n_{2}-4\right)\right]-4 q_{2}^{2} s\left(n_{1}-2, n_{2}\right)\right) \\
& -\quad \frac{\delta_{i_{1}, i_{2}}}{4(d-1) q_{2}^{2}}\left(q_{2}^{2}\left[\left(4 M_{\pi}^{2}+q_{2}^{2}\right) s\left(n_{1}, n_{2}\right)-2\left(s\left(n_{1}-2, n_{2}\right)+s\left(n_{1}, n_{2}-2\right)\right)\right]+s\left(n_{1}-4, n_{2}\right)\right. \\
& \left.-2 s\left(n_{1}-2, n_{2}-2\right)+s\left(n_{1}, n_{2}-4\right)\right), \\
& \mu^{3-d} \int \frac{d^{d} l}{(2 \pi)^{d}} \frac{l_{i_{1}}}{\omega_{l}^{n_{1}} \omega_{\vec{l}+\vec{q}_{2}}^{n_{2}}}=-\frac{\left(q_{2}\right)_{i_{1}}}{2 q_{2}^{2}}\left(q_{2}^{2} s\left(n_{1}, n_{2}\right)+s\left(n_{1}-2, n_{2}\right)-s\left(n_{1}, n_{2}-2\right)\right),
\end{aligned}
$$

with the scalar integrals defined by

$$
s\left(n_{1}, n_{2}\right)=\mu^{3-d} \int \frac{d^{d} l}{(2 \pi)^{d}} \frac{1}{\omega_{l}^{n_{1}} \omega_{\vec{l}+\vec{q}_{2}}^{n_{2}}} .
$$

where $\mu$ denotes the scale of dimensional regularization. The scalar integrals can be further simplified. First, if both of the indices $n_{1}$ and $n_{2}$ are negative or zero, the function $s\left(n_{1}, n_{2}\right)$ vanishes in dimensional regularization:

$$
s\left(n_{1}, n_{2}\right)=0 \text { for } n_{1} \leq 0 \& n_{2} \leq 0
$$

Further, the scalar integrals are symmetric

$$
s\left(n_{1}, n_{2}\right)=s\left(n_{2}, n_{1}\right)
$$

as follows trivially from the substitution $\vec{l} \rightarrow-\vec{l}-\vec{q}_{2}$. If one of the indices $n_{1}$ or $n_{2}$ equals zero, the tensor integrals can be simplified to

$$
\begin{aligned}
& \int \frac{d^{d} l}{(2 \pi)^{d}} \frac{l_{i_{1}} l_{i_{2}} l_{i_{3}}}{\omega_{l}^{n_{1}}}=\int \frac{d^{d} l}{(2 \pi)^{d}} \frac{l_{i_{1}}}{\omega_{l}^{n_{1}}}=0, \\
& \mu^{3-d} \int \frac{d^{d} l}{(2 \pi)^{d}} \frac{l_{i_{1}} l_{i_{2}}}{\omega_{l}^{n_{1}}}=\frac{\delta_{i_{1}, i_{2}}}{d}\left(s\left(n_{1}-2,0\right)-M_{\pi}^{2} s\left(n_{1}, 0\right)\right) .
\end{aligned}
$$

For negative indices, in particular $-5<n_{2}<0$, we can use Eq. (28) to reduce the indices to zero. The scalar integrals of this kind needed for this calculation are given by

$$
\begin{aligned}
& s(4,-4)=\frac{q_{2}^{2}}{d}\left(s(4,0)\left(d q_{2}^{2}-4 M_{\pi}^{2}\right)+2(d+2) s(2,0)\right), \\
& s(4,-2)=q_{2}^{2} s(4,0)+s(2,0) .
\end{aligned}
$$

To further reduce the scalar integrals we use the partial integration technique

$$
\int \frac{d^{d} l}{(2 \pi)^{d}} \frac{\partial}{\partial \vec{l}} \cdot \vec{X} \frac{1}{\omega_{l}^{n_{1}} \omega_{\vec{l}+\vec{q}_{2}}^{n_{2}}}=0
$$

where $\vec{X}$ represents $\vec{l}$ or $\vec{q}_{2}$. Eq. 31 leads to the following reduction formula for the scalar integrals:

$$
\begin{aligned}
s\left(n_{1}, n_{2}\right) & =\frac{1}{\left(n_{2}-2\right) q_{2}^{2}\left(4 M_{\pi}^{2}+q_{2}^{2}\right)}\left(s\left(n_{1}, n_{2}-2\right)\left(q_{2}^{2}\left(-2 d+2 n_{1}+n_{2}-2\right)+2 M_{\pi}^{2}\left(n_{1}-n_{2}+2\right)\right)\right. \\
& \left.+\left(n_{2}-2\right)\left(2 M_{\pi}^{2}+q_{2}^{2}\right) s\left(n_{1}-2, n_{2}\right)-2 M_{\pi}^{2} n_{1} s\left(n_{1}+2, n_{2}-4\right)\right)
\end{aligned}
$$




$$
s\left(n_{1}, 0\right)=-\frac{\left(d-n_{1}+2\right) s\left(n_{1}-2,0\right)}{M_{\pi}^{2}\left(n_{1}-2\right)} .
$$

Eqs. (32) and 33 are used to express all scalar integrals in 3 dimensions in terms of

$$
s(2,0)=-\frac{M_{\pi}}{4 \pi}, \quad s(2,2)=\frac{A\left(q_{2}\right)}{2 \pi},
$$

where

$$
A\left(q_{2}\right)=\frac{1}{2 q_{2}} \arctan \left(\frac{q_{2}}{2 M_{\pi}}\right)
$$

Having performed the Passarino-Veltman reduction as described above, the expressions for box-diagrams of Fig. 1 in $d=3$ dimensions simplify to

$$
\begin{aligned}
& \overrightarrow{\mathcal{A}}_{\mathrm{box}(1)}^{a}=\frac{g_{A}^{5}}{512 \pi F_{\pi}^{4}}\left(2 i\left[\tau_{1} \times \tau_{2}\right]^{a}-3 \tau_{1}^{a}+2 \tau_{2}^{a}\right)\left\{-A\left(q_{2}\right)\left(q_{2}^{2}\left(\vec{\sigma}_{2}+i \vec{\sigma}_{1} \times \vec{\sigma}_{2}\right)+i \vec{q}_{2} \cdot \vec{\sigma}_{2} \vec{q}_{2} \times \vec{\sigma}_{1}\right)\right. \\
& \left.-\left(3 M_{\pi}+2 A\left(q_{2}\right)\left(2 M_{\pi}^{2}+q_{2}^{2}\right)-\frac{M_{\pi}^{3}}{4 M_{\pi}^{2}+q_{2}^{2}}\right) \vec{\sigma}_{1}+A\left(q_{2}\right) \vec{q}_{2} \cdot \vec{\sigma}_{2} \vec{q}_{2}\right\}, \\
& \overrightarrow{\mathcal{A}}_{\text {box }(2)}^{a}=\frac{g_{A}^{5}}{512 \pi F_{\pi}^{4}}\left(2 \tau_{2}^{a}-\tau_{1}^{a}\right)\left\{\left(3 M_{\pi}+q_{2}^{2} A\left(q_{2}\right)-\frac{2 M_{\pi}^{3}}{4 M_{\pi}^{2}+q_{2}^{2}}\right) \vec{\sigma}_{1}+2 q_{2}^{2} A\left(q_{2}\right) \vec{\sigma}_{2}\right. \\
& \left.+\overrightarrow{q_{2}}\left(\left[\frac{A\left(q_{2}\right)\left(8 M_{\pi}^{2}+q_{2}^{2}\right)-2 M_{\pi}}{q_{2}^{2}}-\frac{M_{\pi}}{4 M_{\pi}^{2}+q_{2}^{2}}\right] \vec{q}_{2} \cdot \vec{\sigma}_{1}-2 A\left(q_{2}\right) \vec{q}_{2} \cdot \vec{\sigma}_{2}\right)\right\} \\
& \overrightarrow{\mathcal{A}}_{\text {box }(3)}^{a}=\frac{g_{A}^{5}}{512 \pi F_{\pi}^{4}}\left(2 i\left[\tau_{1} \times \tau_{2}\right]^{a}+3 \tau_{1}^{a}-2 \tau_{2}^{a}\right)\left\{A\left(q_{2}\right)\left(q_{2}^{2}\left(\vec{\sigma}_{2}-i \vec{\sigma}_{1} \times \vec{\sigma}_{2}\right)-i \vec{q}_{2} \cdot \vec{\sigma}_{2} \vec{q}_{2} \times \vec{\sigma}_{1}\right)\right. \\
& \left.+\left(3 M_{\pi}+2 A\left(q_{2}\right)\left(2 M_{\pi}^{2}+q_{2}^{2}\right)-\frac{M_{\pi}^{3}}{4 M_{\pi}^{2}+q_{2}^{2}}\right) \vec{\sigma}_{1}-A\left(q_{2}\right) \vec{q}_{2} \cdot \vec{\sigma}_{2} \vec{q}_{2}\right\}, \\
& \overrightarrow{\mathcal{A}}_{\text {box }(4)}^{a}=\frac{g_{A}^{5}}{512 \pi F_{\pi}^{4}}\left(2 i\left[\tau_{1} \times \tau_{2}\right]^{a}+3 \tau_{1}^{a}+2 \tau_{2}^{a}\right)\left\{A\left(q_{2}\right)\left(q_{2}^{2}\left(\vec{\sigma}_{2}+i \vec{\sigma}_{1} \times \vec{\sigma}_{2}\right)+i \vec{q}_{2} \cdot \vec{\sigma}_{2} \vec{q}_{2} \times \vec{\sigma}_{1}\right)\right. \\
& \left.-\left(3 M_{\pi}+2 A\left(q_{2}\right)\left(2 M_{\pi}^{2}+q_{2}^{2}\right)-\frac{M_{\pi}^{3}}{4 M_{\pi}^{2}+q_{2}^{2}}\right) \vec{\sigma}_{1}-A\left(q_{2}\right) \vec{q}_{2} \cdot \vec{\sigma}_{2} \vec{q}_{2}\right\}, \\
& \overrightarrow{\mathcal{A}}_{\mathrm{box}(5)}^{a}=\frac{g_{A}^{5}}{512 \pi F_{\pi}^{4}}\left(2 \tau_{2}^{a}+\tau_{1}^{a}\right)\left\{\left(3 M_{\pi}+q_{2}^{2} A\left(q_{2}\right)-\frac{2 M_{\pi}^{3}}{4 M_{\pi}^{2}+q_{2}^{2}}\right) \vec{\sigma}_{1}-2 q_{2}^{2} A\left(q_{2}\right) \vec{\sigma}_{2}\right. \\
& \left.+\overrightarrow{q_{2}}\left(\left[\frac{A\left(q_{2}\right)\left(8 M_{\pi}^{2}+q_{2}^{2}\right)-2 M_{\pi}}{q_{2}^{2}}-\frac{M_{\pi}}{4 M_{\pi}^{2}+q_{2}^{2}}\right] \vec{q}_{2} \cdot \vec{\sigma}_{1}+2 A\left(q_{2}\right) \vec{q}_{2} \cdot \vec{\sigma}_{2}\right)\right\} \\
& \overrightarrow{\mathcal{A}}_{\text {box }(6)}^{a}=\frac{g_{A}^{5}}{512 \pi F_{\pi}^{4}}\left(2 i\left[\tau_{1} \times \tau_{2}\right]^{a}-3 \tau_{1}^{a}-2 \tau_{2}^{a}\right)\left\{-A\left(q_{2}\right)\left(q_{2}^{2}\left(\vec{\sigma}_{2}-i \vec{\sigma}_{1} \times \vec{\sigma}_{2}\right)-i \vec{q}_{2} \cdot \vec{\sigma}_{2} \vec{q}_{2} \times \vec{\sigma}_{1}\right)\right. \\
& \left.+\left(3 M_{\pi}+2 A\left(q_{2}\right)\left(2 M_{\pi}^{2}+q_{2}^{2}\right)-\frac{M_{\pi}^{3}}{4 M_{\pi}^{2}+q_{2}^{2}}\right) \vec{\sigma}_{1}+A\left(q_{2}\right) \vec{q}_{2} \cdot \vec{\sigma}_{2} \vec{q}_{2}\right\} .
\end{aligned}
$$

Our final result for the sum of all six box diagrams takes the form

$$
\begin{aligned}
\overrightarrow{\mathcal{A}}_{\mathrm{box}}^{a} & =\frac{g_{A}^{5}}{128 \pi F_{\pi}^{4}}\left\{\tau_{2}^{a}\left[\vec{q}_{2} \cdot \vec{\sigma}_{1} \vec{q}_{2}\left(\frac{\left(8 M_{\pi}^{2}+q_{2}^{2}\right) A\left(q_{2}\right)-2 M_{\pi}}{q_{2}^{2}}-\frac{M_{\pi}}{4 M_{\pi}^{2}+q_{2}^{2}}\right)-\vec{\sigma}_{1}\left(\left(8 M_{\pi}^{2}+3 q_{2}^{2}\right) A\left(q_{2}\right)+3 M_{\pi}\right)\right]\right. \\
& \left.+2 \tau_{1}^{a}\left(\vec{\sigma}_{2} q_{2}^{2}-\vec{q}_{2} \cdot \vec{\sigma}_{2} \vec{q}_{2}\right) A\left(q_{2}\right)\right\}+(1 \leftrightarrow 2),
\end{aligned}
$$

with $q_{2} \equiv\left|\vec{q}_{2}\right|$. This expression coincides with the $g_{A}^{5}$-terms in Eqs. (5.29) and (5.31) of our paper [2] when we set $\vec{k}=0$ to switch off all pion-pole contributions, but differs from the corresponding term in Eq. (7.5) of Ref. [3]. 


\section{SUMMARY AND CONCLUSIONS}

In this paper we have re-derived the non-pion-pole box-diagram contribution to the two-pion-exchange axial-vector current operator proportional to $g_{A}^{5}$ using the approach of the JLab-Pisa group [3] [5]. We have shown at the Fock-space level that the axial-vector currents, constructed using the method of unitary transformation [2] and the time-ordered perturbation theory approach of Ref. [3], are unitary equivalent. The off-shell conditions employed by the JLab-Pisa group are found to correspond to the following choice of the unitary phases

$$
\alpha_{i}^{a x}=0, \quad \alpha_{1}=-\frac{1}{2}, \quad \alpha_{2}=\frac{1}{4},
$$

in the notation of Ref. 2]. Although the phases $\alpha_{i}^{a x}$ are chosen differently by our group in order to enforce renormalizability of the current operators, the two-pion-exchange contributions proportional to $g_{A}^{5}$ are unaffected by this choice $^{5}$ and should therefore coincide in both approaches. We have presented a detailed calculation of the box diagrams starting from the corresponding Fock-space operators and including intermediate steps, thereby reproducing our original result from Ref. [2]. While we have not been able to identify the origin of the disagreement between the two results, see Refs. 2, 14, we hope that our work will help to resolve this issue in the future.

\section{Acknowledgments}

This work is supported in part by the DFG (Grant No. TRR110) and the NSFC (Grant No. 11621131001) through the funds provided to the Sino-German CRC 110 "Symmetries and the Emergence of Structure in QCD", by the BMBF (Grant No. 05P18PCFP1), by the Chinese Academy of Sciences (CAS) President's International Fellowship Initiative (PIFI) (grant no. 2018DM0034) and by VolkswagenStiftung (grant no. 93562).

\section{Appendix A: Transfer matrix with time-dependent interaction}

In this appendix we consider the nuclear transfer matrix in the presence of explicitly time-dependent interactions involving external classical sources. We start with the Schrödinger equation

$$
\left(i \frac{\partial}{\partial t}-H_{0}\right)|\Psi(t)\rangle=V(t)|\Psi(t)\rangle .
$$

As usual, we introduce a free retarded Green's function which satisfies

$$
\left(i \frac{\partial}{\partial t}-H_{0}\right) G_{+}\left(t-t^{\prime}\right)=\delta\left(t-t^{\prime}\right) \quad \text { and } \quad G_{+}\left(t-t^{\prime}\right)=0 \quad \text { for } \quad t<t^{\prime}
$$

and is given by

$$
G_{+}\left(t-t^{\prime}\right)=-i \theta\left(t-t^{\prime}\right) \exp \left[-i\left(H_{0}-i \epsilon\right)\left(t-t^{\prime}\right)\right] .
$$

The solution of the Schrödinger equation can be written as

$$
\left|\Psi^{+}(t)\right\rangle=|\phi(t)\rangle+\int_{-\infty}^{\infty} d t^{\prime} G_{+}\left(t-t^{\prime}\right) V\left(t^{\prime}\right)\left|\Psi^{+}\left(t^{\prime}\right)\right\rangle,
$$

with the state $|\phi(t)\rangle$ satisfying the free Schrödinger equation

$$
\left(i \frac{\partial}{\partial t}-H_{0}\right)|\phi(t)\rangle=0
$$

\footnotetext{
${ }^{5}$ The two-pion exchange contributions to the axial current $\propto g_{A}^{5}$ only depend on the phase $\alpha_{1}^{a x}$, which has been set to zero in Ref. [2].
} 
We now take the Fourier transform of Eq. A.4 by multiplying both sides with $e^{i E t}$ and integrating over time:

$$
\left|\tilde{\Psi}^{+}(E)\right\rangle=|\tilde{\phi}(E)\rangle+\int_{-\infty}^{\infty} d t d t^{\prime} e^{i E t} G_{+}\left(t-t^{\prime}\right) V\left(t^{\prime}\right)\left|\Psi^{+}\left(t^{\prime}\right)\right\rangle
$$

where we have defined

$$
\left|\tilde{\Psi}^{+}(E)\right\rangle=\int_{-\infty}^{\infty} d t e^{i E t}\left|\Psi^{+}(t)\right\rangle, \quad|\tilde{\phi}(E)\rangle=\int_{-\infty}^{\infty} d t e^{i E t}|\phi(t)\rangle .
$$

To simplify Eq. A.6), we Fourier-transform the free Green's function $G_{+}$:

$$
\tilde{G}_{+}(E)=\int_{-\infty}^{\infty} d t e^{i E t} G_{+}(t)=-i \int_{0}^{\infty} d t e^{i\left(E-H_{0}+i \epsilon\right) t}=\frac{1}{E-H_{0}+i \epsilon} .
$$

The backwards transformations are given by

$$
G_{+}(t)=\int \frac{d E}{2 \pi} e^{-i E t} \tilde{G}_{+}(E), \quad\left|\Psi^{+}(t)\right\rangle=\int \frac{d E}{2 \pi} e^{-i E t}\left|\tilde{\Psi}^{+}(E)\right\rangle
$$

Using Eq. A.9), we can rewrite Eq. A.6] into

$$
\begin{aligned}
\left|\tilde{\Psi}^{+}(E)\right\rangle & =|\tilde{\phi}(E)\rangle+\tilde{G}_{+}(E) \int \frac{d E^{\prime}}{2 \pi} \tilde{V}\left(E-E^{\prime}\right)\left|\tilde{\Psi}^{+}\left(E^{\prime}\right)\right\rangle \\
& =|\tilde{\phi}(E)\rangle+\tilde{G}_{+}(E) \int \frac{d E^{\prime}}{2 \pi} T\left(E, E^{\prime}\right)\left|\phi\left(E^{\prime}\right)\right\rangle .
\end{aligned}
$$

Equation A.11 defines the transfer matrix in the presence of an external source. It satisfies the integral equation

$$
T\left(E, E^{\prime}\right)=\tilde{V}\left(E-E^{\prime}\right)+\int \frac{d E^{\prime \prime}}{2 \pi} \tilde{V}\left(E-E^{\prime \prime}\right) \tilde{G}_{+}\left(E^{\prime \prime}\right) T\left(E^{\prime \prime}, E^{\prime}\right)
$$

which can also be written in the equivalent form

$$
T\left(E, E^{\prime}\right)=\tilde{V}\left(E-E^{\prime}\right)+\int \frac{d E^{\prime \prime}}{2 \pi} T\left(E, E^{\prime \prime}\right) \tilde{G}_{+}\left(E^{\prime \prime}\right) \tilde{V}\left(E^{\prime \prime}-E^{\prime}\right) .
$$

Rewriting Eq. A.13 into

$$
\int \frac{d E^{\prime \prime}}{2 \pi} T\left(E, E^{\prime \prime}\right)\left(2 \pi \delta\left(E^{\prime \prime}-E^{\prime}\right)-\tilde{G}_{+}\left(E^{\prime \prime}\right) \tilde{V}\left(E^{\prime \prime}-E^{\prime}\right)\right)=\tilde{V}\left(E-E^{\prime}\right),
$$

and replacing $\tilde{V}\left(E-E^{\prime}\right)$ in Eq. A.10 by left hand side of Eq. A.14 we obtain

$$
\left|\tilde{\Psi}^{+}(E)\right\rangle=|\tilde{\phi}(E)\rangle+\tilde{G}_{+}(E) \int \frac{d E^{\prime}}{2 \pi} \int \frac{d E^{\prime \prime}}{2 \pi} T\left(E, E^{\prime \prime}\right)\left(2 \pi \delta\left(E^{\prime \prime}-E^{\prime}\right)-\tilde{G}_{+}\left(E^{\prime \prime}\right) \tilde{V}\left(E^{\prime \prime}-E^{\prime}\right)\right)\left|\tilde{\Psi}^{+}\left(E^{\prime}\right)\right\rangle .
$$

Using

$$
\int \frac{d E^{\prime}}{2 \pi}\left(2 \pi \delta\left(E^{\prime \prime}-E^{\prime}\right)-\tilde{G}_{+}\left(E^{\prime \prime}\right) \tilde{V}\left(E^{\prime \prime}-E^{\prime}\right)\right)\left|\tilde{\Psi}^{+}\left(E^{\prime}\right)\right\rangle=\left|\tilde{\phi}\left(E^{\prime \prime}\right)\right\rangle
$$

we indeed obtain Eq. A.11.

As a next step, we decompose the interaction into the time-dependent and time-independent parts

$$
V\left(E-E^{\prime}\right)=2 \pi \delta\left(E-E^{\prime}\right) v+v_{5}\left(E-E^{\prime}\right),
$$

where $v$ denotes the time-independent nuclear potential while $v_{5}$ is the part of the interaction that depends on the external axial-vector source. In a similar way, the transfer matrix can be decomposed as

$$
T\left(E, E^{\prime}\right)=2 \pi \delta\left(E-E^{\prime}\right) t(E)+t_{5}\left(E, E^{\prime}\right) .
$$


The off-shell transfer matrix $t(E)$ satisfies the usual Lippmann-Schwinger equation

$$
t(E)=v+v \tilde{G}_{+}(E) t(E)
$$

while the transfer matrix $t_{5}$ fulfills the integral equation

$$
\begin{aligned}
t_{5}\left(E, E^{\prime}\right) & =v_{5}\left(E-E^{\prime}\right)+\tilde{v} G_{+}(E) t_{5}\left(E, E^{\prime}\right)+v_{5}\left(E-E^{\prime}\right) \tilde{G}_{+}\left(E^{\prime}\right) t\left(E^{\prime}\right) \\
& +\int \frac{d E^{\prime \prime}}{2 \pi} v_{5}\left(E-E^{\prime \prime}\right) \tilde{G}_{+}\left(E^{\prime \prime}\right) t_{5}\left(E^{\prime \prime}, E^{\prime}\right) .
\end{aligned}
$$

The last term contributes only to processes with at least two external sources and is, therefore, not considered in our calculation. Taking into account only processes with at most a single insertion of the external sources, we obtain for the transfer matrix

$$
\begin{aligned}
\left(1-v \tilde{G}_{+}(E)\right) t_{5}\left(E, E^{\prime}\right) & =v_{5}\left(E-E^{\prime}\right)\left(1+\tilde{G}_{+}\left(E^{\prime}\right) t\left(E^{\prime}\right)\right) \\
& =v_{5}\left(E-E^{\prime}\right)\left(1+\tilde{G}_{+}\left(E^{\prime}\right) v\left(1-\tilde{G}_{+}\left(E^{\prime}\right) v\right)^{-1}\right) \\
& =v_{5}\left(E-E^{\prime}\right)\left(1-\tilde{G}_{+}\left(E^{\prime}\right) v+\tilde{G}_{+}\left(E^{\prime}\right) v\right)\left(1-\tilde{G}_{+}\left(E^{\prime}\right) v\right)^{-1} \\
& =v_{5}\left(E-E^{\prime}\right)\left(1-\tilde{G}_{+}\left(E^{\prime}\right) v\right)^{-1}
\end{aligned}
$$

which leads to the final expression

$$
t_{5}\left(E, E^{\prime}\right)=\left(1-v \tilde{G}_{+}(E)\right)^{-1} v_{5}\left(E-E^{\prime}\right)\left(1-\tilde{G}_{+}\left(E^{\prime}\right) v\right)^{-1}
$$

One observes, in particular, that all energies in the operator on the left-hand (right-hand) side of $v_{5}$ correspond to the final (initial) state energies. Last but not least, we emphasize that the derived integral equations also follow from the well-known two-potential formalism in scattering theory.

\section{Appendix B: Fock-space expressions for the axial-vector operator}

In this appendix we give the expressions for the axial-vector current operator in Fock space. The corresponding Mathematica notebook is available upon request from the authors. As explained in section II, the axial-vector current $v_{5}^{(n)}$ at the chiral order $Q^{n}$ is obtained following the approach or Refs. [3, 4, by inverting Eq. (12) for the transfer matrix. We find the following results at the first four orders:

$$
\begin{aligned}
v_{5}^{(-3)} & =\frac{\eta A_{N} \eta}{2}-\eta A_{\pi} \frac{\lambda^{1}}{\omega} V \eta+\text { h.c. }, \\
v_{5}^{(-2)} & =\eta A_{\pi} \mathcal{E} \frac{\lambda^{1}}{\omega^{2}} V \eta-\eta H_{0} \eta V \frac{\lambda^{1}}{\omega^{2}} A_{\pi} \eta+\text { h.c. }, \\
v_{5}^{(-1)} & =2 \eta H_{0} \eta V \mathcal{E} \frac{\lambda^{1}}{\omega^{3}} A_{\pi} \eta-\eta H_{0}^{2} \eta V \frac{\lambda^{1}}{\omega^{3}} A_{\pi} \eta-\frac{1}{2} \eta A_{N} \eta V \frac{\lambda^{1}}{\omega^{2}} V \eta+\frac{1}{2} \eta V \frac{\lambda^{1}}{\omega} A_{N} \frac{\lambda^{1}}{\omega} V \eta-\eta A_{\pi} \mathcal{E}^{2} \frac{\lambda^{1}}{\omega^{3}} V \eta \\
& +\eta A_{\pi} \frac{\lambda^{1}}{\omega^{2}} V \eta V \frac{\lambda^{1}}{\omega} V \eta+\frac{1}{2} \eta A_{\pi} \frac{\lambda^{1}}{\omega} V \eta V \frac{\lambda^{1}}{\omega^{2}} V \eta-\eta A_{\pi} \frac{\lambda^{1}}{\omega} V \frac{\lambda^{2}}{\omega} V \frac{\lambda^{1}}{\omega} V \eta+\frac{1}{2} \eta V \frac{\lambda^{1}}{\omega^{2}} V \eta A_{\pi} \frac{\lambda^{1}}{\omega} V \eta \\
& \left.-\eta V \frac{\lambda^{1}}{\omega} A_{\pi} \frac{\lambda^{2}}{\omega} V \frac{\lambda^{1}}{\omega} V \eta+\text { h.c. }, 1\right) \\
v_{5}^{(0)} & =-\frac{1}{2} \eta H_{0} \eta V \frac{\lambda^{1}}{\omega^{3}} V \eta A_{N} \eta+\eta H_{0} \eta V \frac{\lambda^{1}}{\omega^{2}} A_{N} \frac{\lambda^{1}}{\omega} V \eta-\frac{1}{2} \eta A_{N} \eta H_{0} \eta V \frac{\lambda^{1}}{\omega^{3}} V \eta+\frac{1}{2} \eta H_{0} \eta V \frac{\lambda^{1}}{\omega^{3}} V \eta A_{\pi} \frac{\lambda^{1}}{\omega} V \eta \\
& +\frac{1}{2} \eta H_{0} \eta V \frac{\lambda^{1}}{\omega^{3}} V \eta V \frac{\lambda^{1}}{\omega} A_{\pi} \eta+\frac{1}{2} \eta H_{0} \eta V \frac{\lambda^{1}}{\omega^{2}} A_{\pi} \eta V \frac{\lambda^{1}}{\omega^{2}} V \eta-\eta H_{0} \eta V \frac{\lambda^{1}}{\omega^{2}} A_{\pi} \frac{\lambda^{2}}{\omega} V \frac{\lambda^{1}}{\omega} V \eta+\eta H_{0} \eta V \frac{\lambda^{1}}{\omega^{2}} V \eta V \frac{\lambda^{1}}{\omega^{2}} A_{\pi} \eta \\
& -\eta H_{0} \eta V \frac{\lambda^{1}}{\omega^{2}} V \frac{\lambda^{2}}{\omega} A_{\pi} \frac{\lambda^{1}}{\omega} V \eta-\eta H_{0} \eta V \frac{\lambda^{1}}{\omega^{2}} V \frac{\lambda^{2}}{\omega} V \frac{\lambda^{1}}{\omega} A_{\pi} \eta+\eta H_{0} \eta V \frac{\lambda^{1}}{\omega} V \eta V \frac{\lambda^{1}}{\omega^{3}} A_{\pi} \eta-\eta H_{0} \eta V \frac{\lambda^{1}}{\omega} V \frac{\lambda^{2}}{\omega^{2}} A_{\pi} \frac{\lambda^{1}}{\omega} V \eta \\
& -\eta H_{0} \eta V \frac{\lambda^{1}}{\omega} V \frac{\lambda^{2}}{\omega^{2}} V \frac{\lambda^{1}}{\omega} A_{\pi} \eta-\eta H_{0} \eta V \frac{\lambda^{1}}{\omega} V \frac{\lambda^{2}}{\omega} V \frac{\lambda^{1}}{\omega^{2}} A_{\pi} \eta+\eta A_{\pi} \frac{\lambda^{1}}{\omega^{3}} V \eta H_{0} \eta V \frac{\lambda^{1}}{\omega} V \eta+\frac{1}{2} \eta A_{\pi} \frac{\lambda^{1}}{\omega^{2}} V \eta H_{0} \eta V \frac{\lambda^{1}}{\omega^{2}} V \eta
\end{aligned}
$$




$$
\begin{aligned}
& +\frac{1}{2} \eta A_{\pi} \frac{\lambda^{1}}{\omega} V \eta H_{0} \eta V \frac{\lambda^{1}}{\omega^{3}} V \eta+\frac{1}{2} \eta V \frac{\lambda^{1}}{\omega^{3}} V \eta H_{0} \eta A_{\pi} \frac{\lambda^{1}}{\omega} V \eta+\eta A_{N} \eta V \mathcal{E} \frac{\lambda^{1}}{\omega^{3}} V \eta-\eta V \mathcal{E} \frac{\lambda^{1}}{\omega^{2}} A_{N} \frac{\lambda^{1}}{\omega} V \eta \\
& -2 \eta A_{\pi} \mathcal{E} \frac{\lambda^{1}}{\omega^{3}} V \eta V \frac{\lambda^{1}}{\omega} V \eta-\frac{1}{2} \eta A_{\pi} \mathcal{E} \frac{\lambda^{1}}{\omega^{2}} V \eta V \frac{\lambda^{1}}{\omega^{2}} V \eta+\eta A_{\pi} \mathcal{E} \frac{\lambda^{1}}{\omega^{2}} V \frac{\lambda^{2}}{\omega} V \frac{\lambda^{1}}{\omega} V \eta-\eta A_{\pi} \frac{\lambda^{1}}{\omega^{2}} V \eta V \mathcal{E} \frac{\lambda^{1}}{\omega^{2}} V \eta \\
& -\eta A_{\pi} \frac{\lambda^{1}}{\omega} V \eta V \mathcal{E} \frac{\lambda^{1}}{\omega^{3}} V \eta+\eta A_{\pi} \frac{\lambda^{1}}{\omega} V \mathcal{E} \frac{\lambda^{2}}{\omega^{2}} V \frac{\lambda^{1}}{\omega} V \eta+\eta A_{\pi} \frac{\lambda^{1}}{\omega} V \frac{\lambda^{2}}{\omega} V \mathcal{E} \frac{\lambda^{1}}{\omega^{2}} V \eta-\eta V \mathcal{E} \frac{\lambda^{1}}{\omega^{3}} V \eta A_{\pi} \frac{\lambda^{1}}{\omega} V \eta \\
& -\frac{1}{2} \eta V \mathcal{E} \frac{\lambda^{1}}{\omega^{2}} A_{\pi} \eta V \frac{\lambda^{1}}{\omega^{2}} V \eta+\eta V \mathcal{E} \frac{\lambda^{1}}{\omega^{2}} A_{\pi} \frac{\lambda^{2}}{\omega} V \frac{\lambda^{1}}{\omega} V \eta+\eta V \mathcal{E} \frac{\lambda^{1}}{\omega^{2}} V \frac{\lambda^{2}}{\omega} A_{\pi} \frac{\lambda^{1}}{\omega} V \eta+\eta V \frac{\lambda^{1}}{\omega} A_{\pi} \mathcal{E} \frac{\lambda^{2}}{\omega^{2}} V \frac{\lambda^{1}}{\omega} V \eta \\
& + \text { h.c. . }
\end{aligned}
$$

Here, $A_{\pi}\left(A_{N} \propto g_{A}\right)$ refer to the lowest-order vertices that describe the coupling of the external axial source to a single pion field (two nucleon fields). In the notation of Ref. [2], $A_{N}$ and $A_{\pi}$ correspond to the Fock-space operators $A_{2,0}^{(0)}$ and $A_{0,1}^{(-1)}$, respectively. Since the expressions for $v_{5}^{(1)}$ are rather lengthy, we retain below only those contributions which are relevant for the box diagrams, $v_{5 \mathrm{~b}}^{(1)}$, i.e. the terms $\propto g_{A}^{5}$. Further, we distinguish between the pion-pole contributions driven by $A_{\pi}, v_{5 \mathrm{~b}, \mathrm{p}}^{(1)}$, and the non-pion-pole terms $v_{5 \mathrm{~b}, \mathrm{np}}^{(1)}$ associated with $A_{N}$ :

$$
v_{5 \mathrm{~b}}^{(1)}=v_{5 \mathrm{~b}, \mathrm{p}}^{(1)}+v_{5 \mathrm{~b}, \mathrm{np}}^{(1)} .
$$

The expressions for the non-pion-pole contributions have the form:

$$
\begin{aligned}
v_{5 \mathrm{~b}, \mathrm{np}}^{(1)} & =\alpha_{1}\left(\eta A_{N} \eta V \frac{\lambda^{1}}{\omega} V \eta V \frac{\lambda^{1}}{\omega^{3}} V \eta-\eta A_{N} \eta V \frac{\lambda^{1}}{\omega^{3}} V \eta V \frac{\lambda^{1}}{\omega} V \eta\right) \\
& +\alpha_{2}\left(\eta A_{N} \eta V \frac{\lambda^{1}}{\omega} V \frac{\lambda^{2}}{\omega} V \frac{\lambda^{1}}{\omega^{2}} V \eta-\eta A_{N} \eta V \frac{\lambda^{1}}{\omega^{2}} V \frac{\lambda^{2}}{\omega} V \frac{\lambda^{1}}{\omega} V \eta\right) \\
& +\frac{1}{2} \eta A_{N} \eta V \frac{\lambda^{1}}{\omega^{3}} V \eta V \frac{\lambda^{1}}{\omega} V \eta+\frac{3}{8} \eta A_{N} \eta V \frac{\lambda^{1}}{\omega^{2}} V \eta V \frac{\lambda^{1}}{\omega^{2}} V \eta-\frac{1}{2} \eta A_{N} \eta V \frac{\lambda^{1}}{\omega^{2}} V \frac{\lambda^{2}}{\omega} V \frac{\lambda^{1}}{\omega} V \eta+\frac{1}{2} \eta A_{N} \eta V \frac{\lambda^{1}}{\omega} V \eta V \frac{\lambda^{1}}{\omega^{3}} V \eta \\
& -\frac{1}{2} \eta A_{N} \eta V \frac{\lambda^{1}}{\omega} V \frac{\lambda^{2}}{\omega^{2}} V \frac{\lambda^{1}}{\omega} V \eta-\frac{1}{2} \eta A_{N} \eta V \frac{\lambda^{1}}{\omega} V \frac{\lambda^{2}}{\omega} V \frac{\lambda^{1}}{\omega^{2}} V \eta+\frac{1}{8} \eta V \frac{\lambda^{1}}{\omega^{2}} V \eta A_{N} \eta V \frac{\lambda^{1}}{\omega^{2}} V \eta \\
& -\frac{1}{2} \eta V \frac{\lambda^{1}}{\omega^{2}} V \eta V \frac{\lambda^{1}}{\omega} A_{N} \frac{\lambda^{1}}{\omega} V \eta-\eta V \frac{\lambda^{1}}{\omega} A_{N} \frac{\lambda^{1}}{\omega^{2}} V \eta V \frac{\lambda^{1}}{\omega} V \eta+\eta V \frac{\lambda^{1}}{\omega} A_{N} \frac{\lambda^{1}}{\omega} V \frac{\lambda^{2}}{\omega} V \frac{\lambda^{1}}{\omega} V \eta \\
& +\frac{1}{2} \eta V \frac{\lambda^{1}}{\omega} V \frac{\lambda^{2}}{\omega} A_{N} \frac{\lambda^{2}}{\omega} V \frac{\lambda^{1}}{\omega} V \eta+\text { h.c. . B.6) }
\end{aligned}
$$

These are the operators needed to derive the expression for the axial vector current in Eq. 42 at the vanishing momentum of the external source. For the sake of completeness, we also give the operators leading the pion-pole contributions:

$$
\begin{aligned}
v_{5 \mathrm{~b}, \mathrm{p}}^{(1)} & =\alpha_{1}\left(\eta A_{\pi} \frac{\lambda^{1}}{\omega} V \eta V \frac{\lambda^{1}}{\omega^{3}} V \eta V \frac{\lambda^{1}}{\omega} V \eta-\eta A_{\pi} \frac{\lambda^{1}}{\omega} V \eta V \frac{\lambda^{1}}{\omega} V \eta V \frac{\lambda^{1}}{\omega^{3}} V \eta-\eta V \frac{\lambda^{1}}{\omega^{3}} V \eta V \frac{\lambda^{1}}{\omega} V \eta A_{\pi} \frac{\lambda^{1}}{\omega} V \eta\right. \\
& \left.+\eta V \frac{\lambda^{1}}{\omega} A_{\pi} \eta V \frac{\lambda^{1}}{\omega^{3}} V \eta V \frac{\lambda^{1}}{\omega} V \eta\right)+\alpha_{2}\left(\eta A_{\pi} \frac{\lambda^{1}}{\omega} V \eta V \frac{\lambda^{1}}{\omega^{2}} V \frac{\lambda^{2}}{\omega} V \frac{\lambda^{1}}{\omega} V \eta-\eta A_{\pi} \frac{\lambda^{1}}{\omega} V \eta V \frac{\lambda^{1}}{\omega} V \frac{\lambda^{2}}{\omega} V \frac{\lambda^{1}}{\omega^{2}} V \eta\right. \\
& \left.-\eta V \frac{\lambda^{1}}{\omega^{2}} V \frac{\lambda^{2}}{\omega} V \frac{\lambda^{1}}{\omega} V \eta A_{\pi} \frac{\lambda^{1}}{\omega} V \eta+\eta V \frac{\lambda^{1}}{\omega} A_{\pi} \eta V \frac{\lambda^{1}}{\omega^{2}} V \frac{\lambda^{2}}{\omega} V \frac{\lambda^{1}}{\omega} V \eta\right)-\eta A_{\pi} \frac{\lambda^{1}}{\omega^{3}} V \eta V \frac{\lambda^{1}}{\omega} V \eta V \frac{\lambda^{1}}{\omega} V \eta \\
& -\eta A_{\pi} \frac{\lambda^{1}}{\omega^{2}} V \eta V \frac{\lambda^{1}}{\omega^{2}} V \eta V \frac{\lambda^{1}}{\omega} V \eta-\frac{1}{2} \eta A_{\pi} \frac{\lambda^{1}}{\omega^{2}} V \eta V \frac{\lambda^{1}}{\omega} V \eta V \frac{\lambda^{1}}{\omega^{2}} V \eta+\eta A_{\pi} \frac{\lambda^{1}}{\omega^{2}} V \eta V \frac{\lambda^{1}}{\omega} V \frac{\lambda^{2}}{\omega} V \frac{\lambda^{1}}{\omega} V \eta \\
& +\eta A_{\pi} \frac{\lambda^{1}}{\omega^{2}} V \frac{\lambda^{2}}{\omega} V \frac{\lambda^{1}}{\omega} V \eta V \frac{\lambda^{1}}{\omega} V \eta-\frac{1}{2} \eta A_{\pi} \frac{\lambda^{1}}{\omega} V \eta V \frac{\lambda^{1}}{\omega^{3}} V \eta V \frac{\lambda^{1}}{\omega} V \eta-\frac{3}{8} \eta A_{\pi} \frac{\lambda^{1}}{\omega} V \eta V \frac{\lambda^{1}}{\omega^{2}} V \eta V \frac{\lambda^{1}}{\omega^{2}} V \eta \\
& +\frac{1}{2} \eta A_{\pi} \frac{\lambda^{1}}{\omega} V \eta V \frac{\lambda^{1}}{\omega^{2}} V \frac{\lambda^{2}}{\omega} V \frac{\lambda^{1}}{\omega} V \eta-\frac{1}{2} \eta A_{\pi} \frac{\lambda^{1}}{\omega} V \eta V \frac{\lambda^{1}}{\omega} V \eta V \frac{\lambda^{1}}{\omega^{3}} V \eta+\frac{1}{2} \eta A_{\pi} \frac{\lambda^{1}}{\omega} V \eta V \frac{\lambda^{1}}{\omega} V \frac{\lambda^{2}}{\omega^{2}} V \frac{\lambda^{1}}{\omega} V \eta \\
& +\frac{1}{2} \eta A_{\pi} \frac{\lambda^{1}}{\omega} V \eta V \frac{\lambda^{1}}{\omega} V \frac{\lambda^{2}}{\omega} V \frac{\lambda^{1}}{\omega^{2}} V \eta+\eta A_{\pi} \frac{\lambda^{1}}{\omega} V \frac{\lambda^{2}}{\omega^{2}} V \frac{\lambda^{1}}{\omega} V \eta V \frac{\lambda^{1}}{\omega} V \eta+\eta A_{\pi} \frac{\lambda^{1}}{\omega} V \frac{\lambda^{2}}{\omega} V \frac{\lambda^{1}}{\omega^{2}} V \eta V \frac{\lambda^{1}}{\omega} V \eta \\
& +\frac{1}{2} \eta A_{\pi} \frac{\lambda^{1}}{\omega} V \frac{\lambda^{2}}{\omega} V \frac{\lambda^{1}}{\omega} V \eta V \frac{\lambda^{1}}{\omega^{2}} V \eta-\eta A_{\pi} \frac{\lambda^{1}}{\omega} V \frac{\lambda^{2}}{\omega} V \frac{\lambda^{1}}{\omega} V \frac{\lambda^{2}}{\omega} V \frac{\lambda^{1}}{\omega} V \eta-\eta A_{\pi} \frac{\lambda^{1}}{\omega} V \frac{\lambda^{2}}{\omega} V \frac{\lambda^{3}}{\omega} V \frac{\lambda^{2}}{\omega} V \frac{\lambda^{1}}{\omega} V \eta
\end{aligned}
$$




$$
\begin{aligned}
& -\frac{1}{2} \eta V \frac{\lambda^{1}}{\omega^{3}} V \eta V \frac{\lambda^{1}}{\omega} V \eta A_{\pi} \frac{\lambda^{1}}{\omega} V \eta-\frac{1}{2} \eta V \frac{\lambda^{1}}{\omega^{2}} V \eta A_{\pi} \frac{\lambda^{1}}{\omega^{2}} V \eta V \frac{\lambda^{1}}{\omega} V \eta-\frac{1}{4} \eta V \frac{\lambda^{1}}{\omega^{2}} V \eta A_{\pi} \frac{\lambda^{1}}{\omega} V \eta V \frac{\lambda^{1}}{\omega^{2}} V \eta \\
& +\frac{1}{2} \eta V \frac{\lambda^{1}}{\omega^{2}} V \eta A_{\pi} \frac{\lambda^{1}}{\omega} V \frac{\lambda^{2}}{\omega} V \frac{\lambda^{1}}{\omega} V \eta-\frac{3}{8} \eta V \frac{\lambda^{1}}{\omega^{2}} V \eta V \frac{\lambda^{1}}{\omega^{2}} V \eta A_{\pi} \frac{\lambda^{1}}{\omega} V \eta+\frac{1}{2} \eta V \frac{\lambda^{1}}{\omega^{2}} V \eta V \frac{\lambda^{1}}{\omega} A_{\pi} \frac{\lambda^{2}}{\omega} V \frac{\lambda^{1}}{\omega} V \eta \\
& +\frac{1}{2} \eta V \frac{\lambda^{1}}{\omega^{2}} V \eta V \frac{\lambda^{1}}{\omega} V \frac{\lambda^{2}}{\omega} A_{\pi} \frac{\lambda^{1}}{\omega} V \eta+\frac{1}{2} \eta V \frac{\lambda^{1}}{\omega^{2}} V \frac{\lambda^{2}}{\omega} V \frac{\lambda^{1}}{\omega} V \eta A_{\pi} \frac{\lambda^{1}}{\omega} V \eta-\frac{1}{2} \eta V \frac{\lambda^{1}}{\omega} A_{\pi} \eta V \frac{\lambda^{1}}{\omega^{3}} V \eta V \frac{\lambda^{1}}{\omega} V \eta \\
& +\frac{1}{2} \eta V \frac{\lambda^{1}}{\omega} A_{\pi} \eta V \frac{\lambda^{1}}{\omega^{2}} V \frac{\lambda^{2}}{\omega} V \frac{\lambda^{1}}{\omega} V \eta+\frac{1}{2} \eta V \frac{\lambda^{1}}{\omega} A_{\pi} \eta V \frac{\lambda^{1}}{\omega} V \frac{\lambda^{2}}{\omega^{2}} V \frac{\lambda^{1}}{\omega} V \eta+\eta V \frac{\lambda^{1}}{\omega} A_{\pi} \frac{\lambda^{2}}{\omega^{2}} V \frac{\lambda^{1}}{\omega} V \eta V \frac{\lambda^{1}}{\omega} V \eta \\
& +\eta V \frac{\lambda^{1}}{\omega} A_{\pi} \frac{\lambda^{2}}{\omega} V \frac{\lambda^{1}}{\omega^{2}} V \eta V \frac{\lambda^{1}}{\omega} V \eta-\eta V \frac{\lambda^{1}}{\omega} A_{\pi} \frac{\lambda^{2}}{\omega} V \frac{\lambda^{1}}{\omega} V \frac{\lambda^{2}}{\omega} V \frac{\lambda^{1}}{\omega} V \eta-\eta V \frac{\lambda^{1}}{\omega} A_{\pi} \frac{\lambda^{2}}{\omega} V \frac{\lambda^{3}}{\omega} V \frac{\lambda^{2}}{\omega} V \frac{\lambda^{1}}{\omega} V \eta \\
& +\eta V \frac{\lambda^{1}}{\omega} V \eta V \frac{\lambda^{1}}{\omega^{2}} A_{\pi} \frac{\lambda^{2}}{\omega} V \frac{\lambda^{1}}{\omega} V \eta-\eta V \frac{\lambda^{1}}{\omega} V \frac{\lambda^{2}}{\omega} A_{\pi} \frac{\lambda^{1}}{\omega} V \frac{\lambda^{2}}{\omega} V \frac{\lambda^{1}}{\omega} V \eta-\eta V \frac{\lambda^{1}}{\omega} V \frac{\lambda^{2}}{\omega} A_{\pi} \frac{\lambda^{3}}{\omega} V \frac{\lambda^{2}}{\omega} V \frac{\lambda^{1}}{\omega} V \eta+\text { h.c. . }
\end{aligned}
$$

[1] T. S. Park, D. P. Min and M. Rho, Phys. Rept. 233, 341 (1993) hep-ph/9301295.

[2] H. Krebs, E. Epelbaum and U.-G. Meißner, Annals Phys. 378, 317 (2017) arXiv:1610.03569 [nucl-th]].

[3] A. Baroni, L. Girlanda, S. Pastore, R. Schiavilla and M. Viviani, Phys. Rev. C 93, no. 1, 015501 (2016) Erratum: [Phys. Rev. C 93, no. 4, 049902 (2016)] Erratum: [Phys. Rev. C 95, no. 5, 059901 (2017)] arXiv:1509.07039 [nucl-th]].

[4] A. Baroni, L. Girlanda, A. Kievsky, L. E. Marcucci, R. Schiavilla and M. Viviani, Phys. Rev. C 94, no. 2, 024003 (2016) Erratum: [Phys. Rev. C 95, no. 5, 059902 (2017)] arXiv:1605.01620 [nucl-th]].

[5] S. Pastore, L. Girlanda, R. Schiavilla and M. Viviani, Phys. Rev. C 84, 024001 (2011) arXiv:1106.4539 [nucl-th]].

[6] T. T. S. Kuo, S. Y. Lee and K. F. Ratcliff, Nucl. Phys. A 176, 65 (1971).

[7] H. Krebs, V. Bernard and U.-G. Meißner, Annals Phys. 316, 160 (2005) nucl-th/0407078.

[8] K. Suzuki, R. Okamoto, Prog. Theor. Phys. 70, 439 (1983).

[9] S. Okubo, Prog. Theor. Phys. 12, 603 (1954).

[10] E. Epelbaum, Eur. Phys. J. A 34, 197 (2007) arXiv:0710.4250 [nucl-th]].

[11] H. Krebs, E. Epelbaum and U.-G. Meißner, Few Body Syst. 60, no. 2, 31 (2019) arXiv:1902.06839 [nucl-th]].

[12] J. L. Friar, Phys. Rev. C 60, 034002 (1999) nucl-th/9901082.

[13] E. Epelbaum, Prog. Part. Nucl. Phys. 57, 654 (2006) nucl-th/0509032.

[14] A. Baroni et al., Phys. Rev. C 98, no. 4, 044003 (2018) arXiv:1806.10245 [nucl-th]]. 